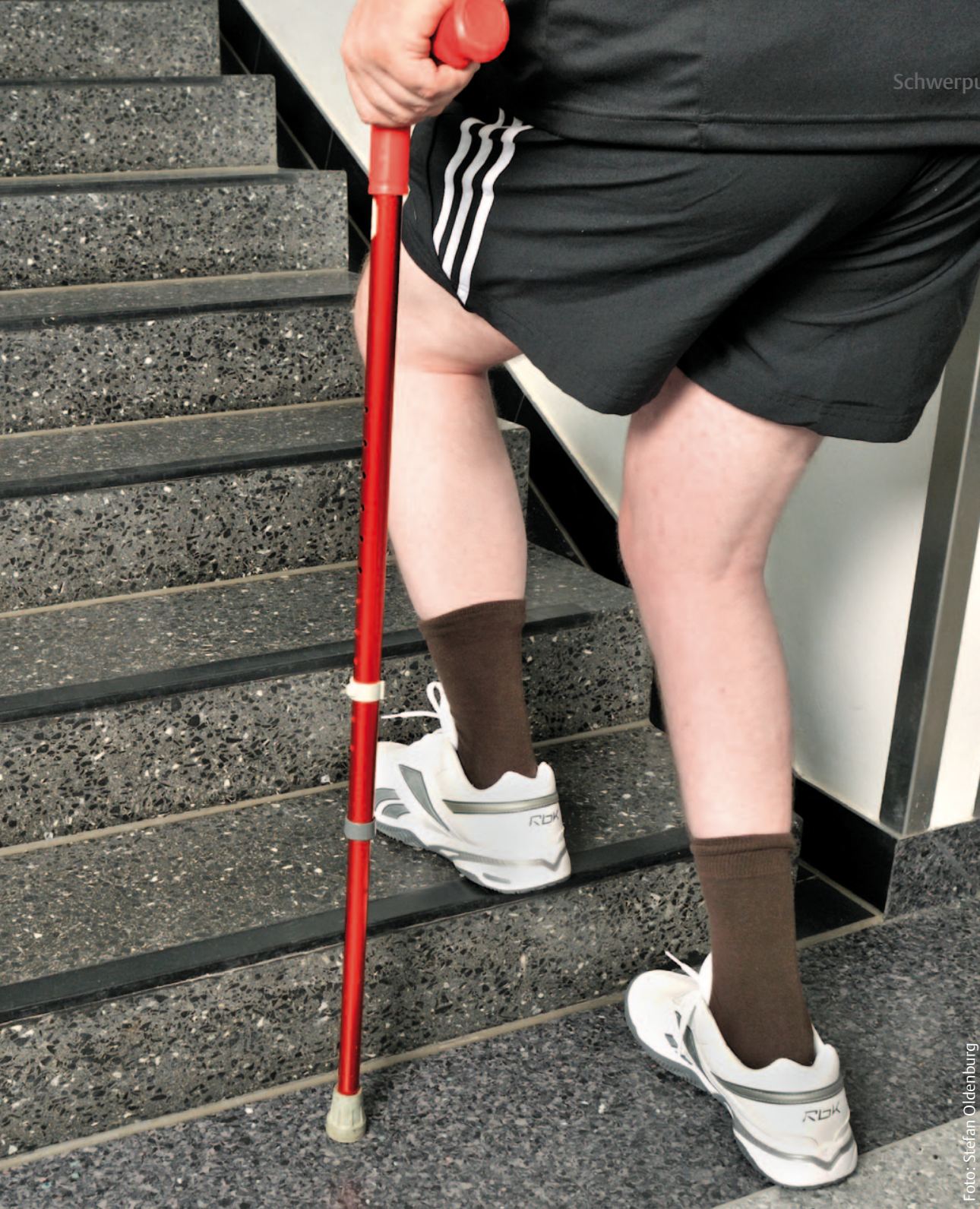

\section{Versorgungsforschung in der Neurorehabilitation - ein Überblick}

Die Versorgungsforschung in Deutschland ist eine junge Disziplin und verglichen mit den USA oder Großbritannien erst im Begriff, sich zu etablieren. Grundlagen schaffte das Gutachten 2001 des Sachverständigenrates für die Konzertierte Aktion im Gesundheitswesen („Über-, Unter- und Fehlversorgung“) - es analysierte die Lage der Gesundheitsversorgung und stellte erste Ergebnisse sowie den Bedarf an Versorgungsforschung dar [46]. Ein Schwerpunktthema war die Versorgung nach Schlaganfall. Bisher dominieren Themen der Akutversorgung die Versorgungsforschungslandschaft - im Bereich rehabilitativer Versorgungsforschung treten neurologische Fragestellungen insgesamt eher in den Hintergrund. Angela Franke, Claudia Wendel und Matthias Morfeld führen in die Grundlagen der Versorgungsforschung ein und verdeutlichen neurorehabilitative Versorgungsforschung an Beispielen. An dieser Stelle sei auch auf den Artikel von Sabine Heel aus 2008 verwiesen - dieser nimmt eine Standortbestimmung der Versorgungsforschung im Bereich Neuropsychologie vor und wurde der vorliegenden Arbeit in weiten Teilen zugrunde gelegt [18].

Angela Franke, Claudia Wendel und Matthias Morfeld

\section{Warum überhaupt Versorgungsforschung?}

Das deutsche Gesundheitssystem steht unter Druck - vor allem die Altersentwicklung der Gesellschaft, die zu mehr Ausgaben und weniger Einnahmen führt, verringert den finanziellen Spielraum im Versorgungssystem. Die Frage, welche Behandlung wann wirklich nötig ist (und welche vielleicht nicht), wird dringlicher. Gleichzeitig hat die ICF das Verständnis von Gesundheit und damit auch von Rehabilitation erweitert [51]. Ziel der Rehabilitation ist nicht mehr nur die Wiederherstellung von Körperfunktionen, sondern ebenfalls die viel schwieriger zu umgrenzende Förderung von Aktivität und Teilhabe. Lösslein bringt die Problematik auf den Punkt: „Lange schon ist klar, dass nicht alles, was medizinisch sinnvoll und machbar ist, auch finanzierbar ist“" [25]. Die Versorgungsforschung soll zur Klärung beitragen, wie die vorhandenen Ressourcen optimal eingesetzt werden können.

Neben finanziellen Begrenzungen verlangen vielfältige Herausforderungen wie z.B. die häufig thematisierte Schnittstellenproblematik oder die Sicherung der Nachhaltigkeit von Leistungen im Bereich der Rehabilitation nach Lösungen.

\section{Was ist Versorgungsforschung?}

Die Versorgungsforschung beschäftigt sich mit der „letzten Meile“ der Kranken- und Gesundheitsversorgung, wie es Pfaff 2003 beschreibt - es geht um das konkrete Versorgungsgeschehen, das in Einrichtungen des Gesundheitssystems mit Patienten stattfindet [32]. In den Blick genommen werden neben medizinischer und psychosozialer Versorgung auch Prävention und Gesundheitsförderung von Individuen und ganzen Populationen. Die Aufgaben der Versorgungsforschung sind in Tab. 1 zusammengefasst.

Forschungsgegenstand ist das Versorgungssystem. Das aus der Systemtheorie stammende Throughput-Modell gliedert den Gegenstandsbereich in einzelne Elemente und dient als strukturierende Forschungsgrundlage ( $\odot$ Abb. 1). Mit den vorhandenen Ressourcen und Rahmenbedingungen (Input) wird in den internen Prozessen des Versorgungssystems (Throughput) die Versorgungsleistung (Output) erzeugt. Daraus resultiert die eigentlich angestrebte Wirkung (Outcome) [33]. Unterschieden werden kann auch hinsichtlich Struktur-, Prozess- und Ergebnisqualität. Der Ergebnisqualität gilt das 


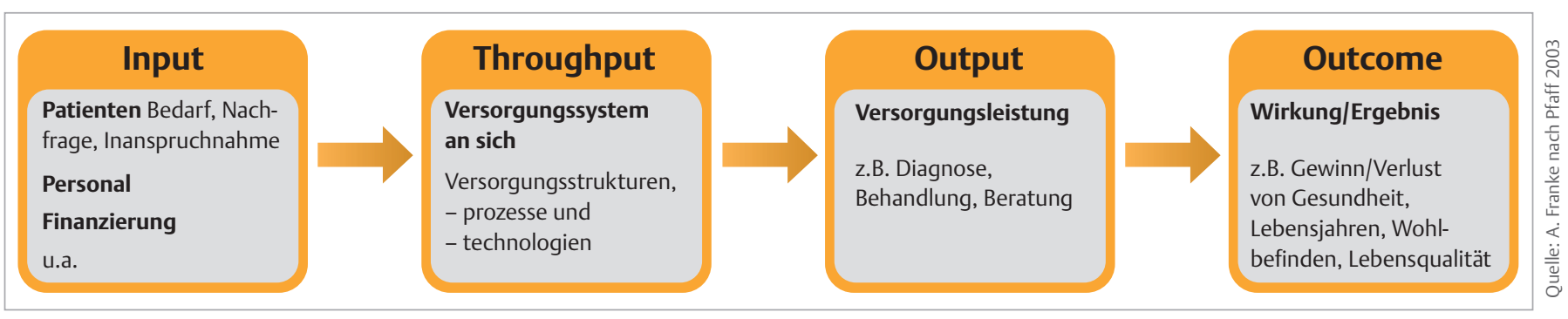

Abb. 1 Das Throughput-Modell nach Pfaff 2003

wesentliche Interesse der Versorgungsforschung, Struktur- und Prozessqualität sind für die Ergebnisqualität von wichtiger Bedeutung [34].

\section{_Was sind die Ziele der Versorgungsforschung?}

Übergeordnetes Ziel ist ein „Optimum an Versorgungsqualität für den einzelnen Patienten“ [43]. Pfaff benennt Patientenorientierung, Qualität und Wirtschaftlichkeit als richtungsweisende Ziele der Versorgungsforschung. Angestrebt wird eine lernende Versorgung - die fortwährende gemeinsame Arbeit der Akteure des Gesundheitssystems an Verbesserungen. Pfaff hebt zudem den integrativen Aspekt der Versorgungsforschung hervor: Sowohl verschiedene Wissenschaftsdisziplinen untereinander als auch Wissenschaft und Praxis müssen Hand in Hand gehen, um die übergeordneten Ziele zu erreichen.

Stärker ausdifferenziert werden die Ziele der Versorgungsforschung in ihren Teildisziplinen. In Tab. 2 sind sieben wichtige Teilgebiete der Versorgungsforschung mit ihren grundlegenden Forschungsfragen dargestellt.

Inwieweit beantwortet die neurorehabilitative Versorgungsforschung die Fragen der einzelnen Teilgebiete bisher und welche Beispiele können benannt werden? Dies sind die Leitfragen der folgenden Abschnitte.

\section{_ Input-Aspekte: Bedarfs- und Inanspruchnahmeforschung} Bedarfsforschung: Ermittlung von subjektivem und objektivem Behandlungsbedarf Ein objektiver Behandlungsbedarf liegt laut Sachverständigenrat für die Konzertierte Aktion im Gesundheitswesen (SVR) vor, wenn folgende Faktoren zusammentreffen [46]:

- der subjektive Bedarf (auch Nachfrage, Wunsch einer Person nach einer Leistung)

- der objektive Bedarf (Feststellung von (drohender) Krankheit oder Behinderung)

- das Vorhandensein geeigneter Behandlungsmöglichkeiten.

\begin{tabular}{|l|l|}
\hline Tab. 1 Die fünf Ebenen der Versorgungsforschung [34] \\
\hline $\begin{array}{l}\text { Aufgaben der } \\
\text { Versorgungsforschung }\end{array}$ & Fragestellung \\
\hline Beschreibung & Wie ist die Versorgung gestaltet? \\
\hline Erklärung & $\begin{array}{l}\text { Welche Ursachen sind für Erfolge oder Probleme der Versorgung } \\
\text { verantwortlich? }\end{array}$ \\
\hline Gestaltung & $\begin{array}{l}\text { Welche Interventionen lassen sich aufgrund versorgungswissen- } \\
\text { schaftlicher Ergebnisse entwickeln (Konzeptentwicklung)? }\end{array}$ \\
\hline Evaluative Begleitung & Welche Implementations- und Umsetzungsprobleme treten auf? \\
\hline Evaluation der Wirksamkeit & Wie wirksam ist die Intervention unter Alltagsbedingungen? \\
\hline
\end{tabular}

Tab. 2 Übersicht über die Teilgebiete der Versorgungsforschung und ihre zentralen Forschungsfragen [33]

\begin{tabular}{|l|l|}
\hline Teilgebiet & Fragestellung \\
\hline Bedarfsforschung & Was ist der Versorgungsbedarf und was sind seine Determinanten? \\
\hline $\begin{array}{l}\text { Inanspruchnahme- } \\
\text { forschung }\end{array}$ & $\begin{array}{l}\text { In welchem Umfang werden Leistungen des Versorgungssystems in } \\
\text { Anspruch genommen und wovon hängt diese Inanspruchnahme ab? }\end{array}$ \\
\hline $\begin{array}{l}\text { Health Technology } \\
\text { Assessment (HTA) }\end{array}$ & $\begin{array}{l}\text { Was sind die Folgen, die mit der Einführung neuer Gesundheit- } \\
\text { stechnologien verbunden waren oder sein werden? }\end{array}$ \\
\hline Organisationsforschung & $\begin{array}{l}\text { Welche Versorgungsstrukturen und -prozesse sind in den } \\
\text { Versorgungsorganisationen wirksam? }\end{array}$ \\
\hline Versorgungsökonomie & $\begin{array}{l}\text { Was sind die Kosten und was ist der Nutzen } \\
\text { einer Versorgungsleistung? }\end{array}$ \\
\hline Qualitätsforschung & $\begin{array}{l}\text { Wie ist die Qualität der Leistungserstellung, des Outputs und } \\
\text { des Outcomes? }\end{array}$ \\
\hline $\begin{array}{l}\text { Versorgungs- } \\
\text { epidemiologie }\end{array}$ & $\begin{array}{l}\text { Wie wirken sich Behandlungsmethoden, Verfahren und Leitlinien } \\
\text { im Alltag aus? }\end{array}$ \\
\hline
\end{tabular}

Diese Taxonomie nimmt eine erste Strukturierung der Versorgungsforschung vor. Die einzelnen Domänen greifen ineinander.

Behandlungsmöglichkeiten konkretisiert der SVR als spezifizierbare Maßnahmen, die gesundheitlichen Nutzen erwarten lassen [46]. Von Nutzen wird gesprochen, wenn die Verbesserung des Gesundheitsstatus das Risiko überwiegt. Was als Bedarf angesehen wird, hängt auch vom gesellschaftlichen Kontext ab: Hier sei nochmals die ICF angeführt, die den vormals funktionell geprägten Behinderungsbegriff um eine Teilhabe-Behinderung erweitert hat.

Nicht immer treffen die drei Kriterien gleichzeitig zusammen, so kann z. B. ein objektiver Bedarf ohne Nachfrage oder ein subjektiver Bedarf ohne objektivierbares Äqui- valent vorkommen. Die Bedarfsforschung beschäftigt sich mit der Ermittlung von subjektivem und objektivem Behandlungsbedarf und ihren Determinanten [46].

Neurorehabilitative Bedarfsforschung Der objektive Bedarf an neurorehabilitativer Behandlung insgesamt kann nicht ohne Weiteres ermittelt werden. Schätzdaten zur Prävalenz neurologischer Erkrankungen liegen zwar vor, jedoch schließt sich an eine Erkrankung nicht immer Rehabilitationsbedarf an. Auch findet eine flächendeckende Erhebung relevanter Erkrankungen bisher nicht standardmäßig statt - gesetzlich veranker- 
te Registerdaten wie z. B. für Krebserkrankungen liegen so nicht vor. Ein aus diesem Problemkreis entstandenes Projekt ist die Einrichtung eines nationalen Schlaganfallregisters. Regionale und einrichtungsbezogene Initiativen schlossen sich 1999 als Arbeitsgemeinschaft Deutscher SchlaganfallRegister (ADSR) zusammen. Die bisherigen Daten stammen vor allem aus dem Akutbereich. Eine Erweiterung der Datensammlung auf den Reha- und Nachsorgebereich wird benötigt und angestrebt [6,19]. Wie ein Register beschaffen sein muss, um optimal für Versorgungsforschungszwecke nutzbar zu sein, stellt das Deutsche Netzwerk Versorgungsforschung (DNVF) in einem aktuellen Memorandum dar [31].

Von den verschiedenen Leistungsträgern erhobene Routinedaten bilden die gegenwärtige Situation der Inanspruchnahme neurologischer Rehabilitation ab. Sie lassen jedoch offen, wo objektiver Bedarf ungedeckt bleibt. Auch zur Bedarfsentwicklung mangelt es an Daten. Noch unklar ist z.B., wie sich durchgeführte Präventionsmaßnahmen auf die Schlaganfall-Inzidenz auswirken [19]. Für die Zuweisung und Genehmigung von Rehabilitationsmaßnahmen ist ein vorliegender Bedarf Voraussetzung, dieser erfordert eine objektive Bedarfsfeststellung [35]. Die Praxis der Bedarfsfeststellung in der Rehabilitation wurde bisher wenig erforscht [50]. Bei der Ermittlung des Behandlungsbedarfs besteht zum Teil Spielraum, z.B. wegen mangelnder Evidenz dahingehend, wann eine Behinderung droht. Mittag und Jäckel äußern Bedenken, dass die Bedarfsfeststellung als „Stellschraube“ beim Einsatz vorhandener Ressourcen genutzt werden könnte [27]. Eine Arbeit von Mühlig et al. verdeutlicht dies für den Bereich der Neurorehabilitation anhand unterschiedlicher Kostenübernahmequoten verschiedener Leistungsträger in Bezug auf eine neuropsychologische Behandlung [28]. Zudem muss für neuropsychologische Behandlung generell eine ausgeprägte Diskrepanz zwischen Angebot (Vorhandensein geeigneter Behandlungsmöglichkeiten) und objektivem Bedarf sowohl im stationären/teilstationären als auch ambulanten Bereich berichtet werden [18].

Ein zusätzlicher Bedarf an Unterstützung ergibt sich aus der Situation, die für das soziale Umfeld neurologisch Erkrankter entsteht. Durch das Krankheitsbild der Betroffenen - gekennzeichnet etwa durch geminderte Kommunikationsfähigkeit, Orientierung und Selbstwahrnehmung - ergeben sich für Angehörige oft immense Anforderungen. Diese Belastungen können bei den unterstützenden Angehörigen negatives Befinden bis hin zu krankheitswertigen psychischen Beeinträchtigungen hervorrufen. Daraus kann ein eigener Behandlungsbedarf entstehen, zudem wird von ungünstigen Rückwirkungen auf die Betroffenen berichtet. Beklagt wird, dass für Angehörige professionelle Unterstützungsangebote weitgehend fehlen, obwohl erfolgreiche Interventionen in diesem Bereich belegt sind [49].

\section{Inanspruchnahmeforschung}

Nicht jeder Behandlungsbedarf führt zu einer Nutzung des Versorgungssystems. Die Inanspruchnahmeforschung untersucht, wann eine Leistung in Anspruch genommen wird und wodurch dies bedingt ist. Die Inanspruchnahme setzt Nachfrage und Zugang zu einer Leistung voraus. Ein Fehlverhältnis zwischen Bedarf und Inanspruchnahme wird als Über- oder Unterversorgung bezeichnet [34]. Nach einem Modell von Andersen sind die wichtigsten Einflussfaktoren auf die Inanspruchnahme des Gesundheitssystems [1]: - Prädisponierende Faktoren (demografisch, sozial, Gesundheitseinstellungen)

- Bedarf (subjektiv, objektiv) und

- Zugangsressourcen (persönlich, systembezogen).

Folgen einer Inanspruchnahme wie z. B. Gesundheitsstatus und Zufriedenheit wirken sich später auf das weitere Inanspruchnahmeverhalten aus. Dieses Modell hat sich auch in der deutschen Inanspruchnahmeforschung durchgesetzt und bewährt [48].

Inanspruchnahme der Neurorehabilitation Als Forschungsgrundlage werden unterschiedlich stark verdichtete Inanspruchnahmedaten von den gesetzlichen Krankenkassen sowie der Deutschen Rentenversicherung zur Verfügung gestellt [42]. Diese Routinedaten sind jedoch für viele Fragestellungen nicht ausreichend. Sie berücksichtigen zu wenig die Heterogenität und Komplexität von Erkrankungen, wie Heuschmann und sein Team bezüglich des Schlaganfalls darstellen [19]. Eine hinreichende Erklärung des Inanspruchnahmeverhaltens ist deshalb mit diesen Daten kaum möglich. Auch die Frage, warum Leistungen nicht in Anspruch genommen werden, können Routinedaten nicht beantworten.

Deskriptiv berichten Heuschmann und seine Kollegen auf Datengrundlage des
Schlaganfallregisters Nordwestdeutschland über die Inanspruchnahme stationärer Rehabilitation nach Schlaganfall. Rund 26\% der Patienten werden nach stationärer Akutbehandlung in eine stationäre Rehaeinrichtung entlassen, rund 57\% nach Hause. Offen bleibt, ob die nach Hause Entlassenen andere Rehabilitationsleistungen in Anspruch nehmen. Die Arbeit von Heuschmann ist nicht auf die Erklärung der Inanspruchnahmedaten ausgerichtet, dennoch zeigt sie, dass die Inanspruchnahme stationärer Rehabilitation mit dem Grad der Selbstständigkeit bei Alltagsaktivitäten, gemessen mit dem BarthelIndex (BI), zusammenhängt. Die Entlassung in stationäre Rehabilitation ist bei geringerer Selbstständigkeit $(\mathrm{BI}<65)$ die häufigste Entlassungsform, die Entlassung nach Hause bei höherer Selbstständigkeit (BI>65). Zur Inanspruchnahme der Rehabilitation nach Schlaganfall werden weitere Daten gefordert [19].

Verschiedene Faktoren beeinflussen Inanspruchnahmeverhalten

Inanspruchnahme von Neurorehabilitation scheint zudem kulturell beeinflusst zu sein, wie eine Arbeit zur stationären Rehabilitation nach Schlaganfall in Taiwan zeigt. Trotz kostenloser staatlicher Gesundheitsversorgung war die Inanspruchnahme mit 34\% vergleichsweise gering. Diskutiert wird, ob dies zumindest teilweise durch größere soziale Unterstützung im persönlichen Umfeld zu erklären ist [24]. Eine amerikanische Studie macht neben familiärer Unterstützung zudem Alter, Geschlecht, Ethnizität, Region, Haushaltseinkommen, Art des Schlaganfalls und die Aufenthaltsdauer in der Akutklinik als Faktoren aus, die eine Inanspruchnahme von Rehabilitationsleistungen nach Schlaganfall beeinflussen [7]. Aufgrund kultureller und gesundheitssystemischer Unterschiede können diese Ergebnisse jedoch nicht ohne Weiteres auf Deutschland übertragen werden. Es bleibt weitgehend offen, welche Faktoren für die Nutzung neurorehabilitativer Leistungen bedeutsam sind.

Zum deutschen Gesundheitssystem nimmt der BARMER GEK Heil- und Hilfsmittel-Report 2010 Stellung. Er berichtet von einer höheren Inanspruchnahme ergotherapeutischer Leistungen in Gebieten mit mehr Ergotherapiepraxen - die Niederlassung von Heilmittelerbringern wie der Ergotherapie wird bisher nicht wie bei den Arztoder Psychotherapiepraxen reguliert. Unklar bleibt, ob die Regionen mit mehr therapeu- 
tischen Angeboten überversorgt sind oder eine ergotherapeutische Unterversorgung in den Gebieten niedrigerer Inanspruchnahme vorliegt [39]. Hier zeigt sich, wie wichtig es ist, Bedarf klar zu definieren.

\section{Throughput-Elemente: interne Prozesse des Versorgungssystems}

Health Technology Assessment (HTA): Hilfe bei Handlungsempfehlungen und Grundlage politischer Entscheidungen Beim HTA werden gesundheitstechnologiebezogene Forschungsergebnisse in systematischen Übersichten zusammengetragen. Diese führen zu Handlungsempfehlungen für die Praxis und können als evidenzbasierte Grundlage politischer Entscheidungen dienen [32]. HTA-Berichte bewerten „Nutzen, Risiko, Kosten und Auswirkungen von Verfahren und Technologien mit Bezug zur gesundheitlichen Versorgung der Bevölkerung“ [10]. Dabei finden neben der experimentellen Wirksamkeit (Efficacy) die Wirksamkeit unter Alltagsbedingungen (Effectiveness), Wirksamkeitsvergleiche (Comparative Effectiveness), eine gesundheitsökonomische Bewertung (Efficiency) und der soziale, rechtliche und ethische Rahmen Berücksichtigung [10].

HTA in der Neurorehabilitation

In Deutschland werden HTA-Berichte unter anderem zentral von der Deutschen Agentur für HTA (DAHTA) des Deutschen Institutes für Medizinische Dokumentation und Information (DIMDI), einer Behörde des Bundesministeriums für Gesundheit, veröffentlicht. Die DAHTA stellt ein datenbankgestütztes Informationssystem mit eigenen und anderen nationalen sowie internationalen Berichten zu gesundheitsrelevanten Verfahren zur Verfügung. Es gibt nur wenige DAHTA-Berichte aus dem Bereich Neurorehabilitation, sie betreffen pflegerische Versorgungskonzepte bei Demenzerkrankungen und kognitives Training bei Demenzen und anderen Störungen mit kognitiven Defiziten. Da bei beiden Berichten nur wenige Studien die Einschlusskriterien bezüglich Methodik, Stichprobengröße und Aktualität erfüllten, konnten keine Praxisempfehlungen abgeleitet werden. Als problematisch schätzen die Forscher neben den methodischen Kriterien der Studien die große Heterogenität und damit mangelnde Vergleichbarkeit der berücksichtigten Wirksamkeitskriterien ein [15, 37].
Auf europäischer Ebene ist ein European Network for Health Technology Assessment im Aufbau (EUnetHTA) [12]. Auch die internationale Cochrane Collaboration stellt HTAInformationen zur Verfügung. Die Cochrane-Arbeiten zum HTA in der Neurorehabilitation befassen sich z.B. mit beruflicher Rehabilitation bei Multipler Sklerose, mit Psychotherapie bei Angststörung nach Schädel-Hirn-Trauma, mit kognitivem Training bei Demenz, mit Informationsvermittlung für Schlaganfallbetroffene und ihre Angehörigen sowie mit Ergotherapie bei kognitiven Beeinträchtigungen nach Schlaganfall $[8,20$, 23, 44, 45]. Auch hier besteht zum Teil das Problem, dass Studien den methodischen Anforderungen nicht genügen und nicht $\mathrm{zu}$ Handlungsempfehlungen führen.

Insbesondere im Heil- und Hilfsmittelbereich, wozu auch Ergo-, Physiotherapie und Logopädie zählen, wird mehr HTA und Versorgungsforschung allgemein gefordert. Dieser für die Rehabilitation wichtige Bereich wird bisher eher vernachlässigt [39]. Trotzdem sind bereits HTA-bezogene Ergebnisse aus dem Heilmittelbereich vorhanden. So kommt z.B. aus dem physiotherapeutischen Spektrum eine Übersichtsarbeit zu „Massed Practice“, einem motorischrehabilitativen Trainingskonzept, das durch hohe Übungsintensität an der individuellen Leistungsgrenze gekennzeichnet ist und auf neuronaler Plastizität beruht [36]. „Massed Practice“ wird in verschiedenen Verfahren bei Hemiparese nach Schlaganfall zum Training der oberen und unteren Extremität angewendet. Renner und Hummelsheim greifen Efficacy, Effectiveness und Comparative Effectiveness unterschiedlicher Verfahren, die das „Massed Practice“-Konzept verwenden, auf und berichten von durchgängig positiven Ergebnissen.

\section{Organisationsforschung}

Die Organisationsforschung beschreibt und analysiert als weiteres Throughput-Element die Strukturen und Prozesse der Versorgungsorganisationen. Darüber hinaus werden auch die Beziehungen zwischen den Organisationen betrachtet, die in ihrer Gesamtheit das Versorgungssystem ausmachen [32].

Organisation der neurologischen Rehabilitation

Organisatorische Besonderheiten der Neurorehabilitation beschreiben Wallesch und Ko- enig [49]. Spezielle Charakteristika auf Prozessebene sind, dass neurologische Rehabilitation sehr früh beginnt, auf schwerst beeinträchtigte Menschen ausgerichtet ist und die Einbeziehung der Angehörigen einen entsprechenden Stellenwert einnimmt. Strukturelle Merkmale sind die Bereitstellung verhältnismäßig vieler Rehabilitationsplätze im Vergleich zu neurologischen Akutbehandlungsbetten und die Einteilung in die Behandlungsphasen A bis F. Phase D entspricht der medizinischen Rehabilitation anderer Indikationen und ist durch ein multiprofessionelles Behandlungsteam, hohe Therapieintensität und Alltagstraining gekennzeichnet. Wichtige Schnittstelle ist das Ende dieser Phase, wo ein Überleitungsmanagement in das häusliche und ggf. berufliche Umfeld stattfinden muss.

Phase D kann stationär oder bei entsprechendem Angebot ambulant stattfinden. Leider wird ambulante neurorehabilitative Komplexbehandlung - obwohl gesetzlich gefordert ( $\$ 40$ SGB V) und 2005 von der Bundesarbeitsgemeinschaft für Rehabilitation empfohlen - nicht flächendeckend angeboten, da eine angemessene Vergütung der Leistungsträger nicht gesichert ist [4]. Auch wenn stationäre Rehabilitationseinrichtungen in der Initialphase der Rehabilitation Strukturvorteile bieten, ist eine ambulantwohnortnahe Behandlung oft besser geeignet, den Alltagstransfer wiedererlangter Kompetenzen zu gewährleisten und das Umfeld der Betroffenen einzubeziehen $[9,25]$. Auch im Anschluss an Phase D treffen die Rehabilitanden häufig auf strukturelle Defizite - sie benötigen noch immer Unterstützungsangebote, insbesondere an neuropsychologischen Weiterbehandlungsmöglichkeiten fehlt es jedoch oft $[18,49]$.

Mehr Organisationsforschung wird in Bezug auf neue Versorgungskonzepte gefordert. Ein Beispiel dafür ist die integrierte Versorgung. Ihr Ziel ist eine bessere Koordination und Zusammenarbeit einzelner Akteure der Versorgung. Im Jahr 2000 eingeführt und immer weiter gefördert, entstand eine bedeutende Anzahl integrierter Verträge - vor allem im Akutbereich. Wie die Projekte der integrierten Versorgung umgesetzt wurden und ob sie ihre Ziele erreichten, ist weitgehend unklar [40]. Obwohl das Konzept integrierter Versorgung auch zum Schnittstellenmanagement in der neurologischen Rehabilitation geeignet erscheint, ist wenig über derartige Projekte bekannt. 
TEMPiS-Konzept bietet ländlichen Regionen in Südostbayern eine optimale Versorgung nach Schlaganfall

Ein umfassend evaluiertes Gegenbeispiel ist das 2003 ins Leben gerufene telemedizinische Pilotprojekt zur integrierten Schlaganfallversorgung in der Region Südostbayern (TEMPiS). Es ist eher dem Akutbereich zuzuordnen, schließt jedoch auch frührehabilitative Elemente ein. Konzept des Projektes ist die Vernetzung von regionalen Krankenhäusern mit spezialisierten Schlaganfallzentren mittels Telemedizin, Weiterbildung und Behandlungsempfehlungen, um auch in ländlichen Regionen eine optimale Schlaganfallversorgung zu ermöglichen. Veränderungen in den teilnehmenden Kliniken nach der Einbindung in das Netzwerk waren z.B. die Einführung von Ergotherapie und Logopädie sowie vermehrte physiotherapeutische Behandlung [29]. Im Ergebnis führte die Behandlung nach dem TEMPiS-Konzept im Vergleich zur Regelbehandlung zu einer deutlich günstigeren Prognose für die Patienten [30].

\section{Versorgungsökonomie: wirtschaftlicher Nutzen der neuen Konzepte}

Im Mittelpunkt der Versorgungsökonomie steht die Finanzierung des Gesundheitssystems. Fragen werden nach der Kosten-Nutzen-Relation von Strukturen, Abläufen und Verfahren und damit nach dem (quantitativen) Verhältnis vom Input zum Output gestellt. Neben materiellen Ressourcen werden auch immaterielle Kosten der Versorgung betrachtet [32].

Versorgungsökonomie der Neurorehabilitation

Grundlagen versorgungsökonomischer Betrachtungen durch eine Konkretisierung der Kostenseite schafft z.B. eine Erhebung direkter Kosten der Parkinson-Behandlung [11]. Die Forscher analysierten direkte medizinische Gesamtkosten pro Quartal sowie Anteile unterschiedlicher Leistungen. Hauptkostenfaktor war die medikamentöse Versorgung, gefolgt von Krankenhausaufenthalten, nichtmedikamentösen Therapien und stationärer Rehabilitation. Nichtmotorische Komplikationen wie Depression, Demenz und Psychosen erhöhten die Gesamtkosten. Einsparpotenzial sehen Ehret und seine Kollegen bei einer stärker leitlinienkonformen Behandlung, ambulanter statt stationärer Leistungen und einer in- tegrierten Versorgung in Hinblick auf die Vermeidung kostenintensiver stationärer Aufenthalte. Hier könnten zukünftige versorgungsökonomische Untersuchungen anknüpfen.

Versorgungsökonomische Aspekte greift auch der BARMER GEK Heil- und Hilfsmittel-Report 2010 auf. Deutlich wird, dass die Ausgaben im Bereich der Heil- und Hilfsmittel zugenommen haben und weiter steigen, insgesamt aber nur einen geringen Teil der Gesamtausgaben der Krankenkassen ausmachen. In manchen Fällen können Heilmittelleistungen wie Physio- oder Ergotherapie eine Arzneimitteltherapie ersetzen und sind sogar kostengünstiger und deshalb auch aus ökonomischer Sicht interessant. Zur vergleichenden Prüfung der Kosten-Nutzen-Relationen - insbesondere unter Beachtung indirekter Kosten wie z.B. Nebenwirkungen - sowie zur evidenzbasierten Beurteilung und Steuerung der Entwicklungen im Heilmittelbereich ist allerdings noch viel Forschungsarbeit notwendig [39].

Ambulante Behandlung effektiver als stationäre bezüglich Alltagsfähigkeiten und Vitalität

Klare Ergebnisse liefert die Arbeit von Bölsche und seinem Team [5]. Sie untersuchten in einer Pilotstudie Outcome-Unterschiede der Phase-D-Rehabilitation nach Schlaganfall in Abhängigkeit von der Form des Behandlungssettings. Die Ergebnisse von ambulanter und stationärer Rehabilitation sind insgesamt vergleichbar, jedoch sind höhere Effekte bezüglich der Alltagsfähigkeiten und Vitalität Vorteile der ambulanten Behandlungsform. Diese Ergebnisse stimmen mit denen von Schönle überein und legen nahe, dass bei geringeren Kosten eine Rehabilitation im ambulanten Setting vorzuziehen ist, wenn keine anderen Gründe dagegensprechen und entsprechende Angebote verfügbar sind [41]. Bölsche und seine Kollegen merken jedoch an, dass die Ergebnisse dieser kleinen Studie $(n=53)$ weiter abgesichert werden müssen [5]. Sie diskutieren außerdem, dass vor allem die Inhalte einer Rehabilitationsmaßnahme, weniger das Setting, das Outcome bestimmen. Auch indirekte Kosten einer ambulanten Maßnahme werden angemerkt: Bei ambulanter Rehabilitation ist die Unterstützung der Angehörigen wesentlich stärker gefragt.
Pay for Performance (P4P) - Entlohnung nach Leistung

Um direkte Kosten geht es bei dem versorgungsökonomischen Konzept Pay for Performance (P4P) - Entlohnung nach Leistung. Dieses aktuell in Deutschland diskutierte Vergütungsprinzip wurde vor einigen Jahren in den USA eingeführt, um damals vorherrschenden, gravierenden Qualitätsproblemen im US-Gesundheitswesen entgegenzuwirken [21]. Hierzulande waren es zudem Fragen der Finanzierung von Gesundheitsversorgung, die zur Erprobung von P4P führten. Die in Deutschland üblichen Vergütungssysteme bergen ungewünschte Anreize in sich: Fallpauschalen können zur Reduktion von Aufenthaltsdauern und Versorgungsleistungen verleiten, Tagessätze zur Verlängerung der Aufenthaltsdauer und ebenfalls zu Leistungseinsparungen. Daher ist schlüssig, dass die Vergütung von Leistungen an der tatsächlichen Zielerreichung (Performance) bemessen werden sollte.

Ob sich mit diesem neuen Konzept tatsächlich Rehabilitationsergebnisse verbessern und/oder die Kosten der Versorgung senken lassen, ist eine Frage, der sich das Modellprojekt „Ergebnisorientierte Vergütung der Rehabilitation nach Schlaganfall“ annimmt. Es wurde 2001 bis 2008 durchgeführt und in einer groß angelegten multizentrischen Studie evaluiert. Mit den finanziellen Anreizen sollte der Qualitätswettbewerb unter den Kliniken gefördert werden. Über Boni für überdurchschnittlich gute und entsprechende Abzüge für weniger gute Kliniken entschied ein wissenschaftliches Institut bei Kostenneutralität für die Leistungsträger. Kritisch ist anzumerken, dass wesentliche Rehabilitationsziele bei der Bewertung außer Acht gelassen wurden. Befürchtet werden muss, dass sich teilnehmende Kliniken nur auf die vergütungsrelevanten Behandlungsaspekte konzentrieren könnten und weiche Outcome-Faktoren wie z.B. Krankheitsverarbeitung sowie die individuellen Prioritäten der Behandelten vernachlässigt würden [16]. Auch die Nachhaltigkeit der Behandlungsergebnisse - obwohl bedeutsam für weitere Behandlungskosten floss nicht in die Untersuchungen ein. Zudem ist grundsätzlich die Gerechtigkeit dieses Vergütungssystems zu hinterfragen: Auch wenn das Leistungsniveau aller Kliniken steigen würde, hätten die „Schlechtesten“ trotz interner Verbesserungen Abzüge hinzunehmen. Somit wird vor allem Wett- 
bewerb erzeugt und nicht unbedingt eine gerechte Belohnung qualitativ hochwertiger, kostensparend ausgerichteter Arbeit („Pay for Performance“) gewährleistet - auch damit könnten ungünstige und ungewollte Anreize gesetzt werden.

\section{_ Outcome-Elemente: Wirkung und Ergebnisse der Versorgung Qualitätsforschung}

Die Qualitätsforschung beschreibt und erklärt Throughput (Struktur- und Prozessqualität), Output und Outcome auf qualitativer Ebene (Ergebnisqualität) [32]. Dazu gehören das Messen von Qualitätsparametern und die Bereitstellung geeigneter Instrumente. Qualitätssicherung und -management ist damit ebenfalls ein Ziel der Versorgungsforschung [52].

Qualitätsforschung in der Neurorehabilitation

Laut §20 SGB IX sind qualitätssichernde Maßnahmen für alle Leistungserbringer der Rehabilitation verpflichtend. Stationäre Rehabilitationseinrichtungen müssen ihr Qualitätsmanagement zudem zertifizieren lassen. Effektivität und Effizienz von Qualitätsmanagement-Programmen sind allerdings aufgrund mangelnder systematischer Untersuchungen noch kaum belegt [22]. Um die praktische Relevanz der Qualitätsdaten zu erhöhen, regen Farin, Jäckel und Schalaster an, klinikbezogene Ergebnisse des Qualitätssicherungsverfahrens allgemein zugänglich zu machen sowie gute Leistungen z.B. mit finanziellen Anreizen oder Belegungsvorteilen zu belohnen [14]. Public Disclosure - die nichtanonymisierte Veröffentlichung von Qualitätsdaten - soll den Qualitätswettbewerb anregen. Eine tendenziell qualitätssteigernde Wirkung gilt als belegt, die Anwendbarkeit im deutschen Gesundheitssystem jedoch als noch zu prüfen [47].

Qualitätsforschung wird auch von Logopäden, Ergo- und Physiotherapeuten im Zuge der zunehmenden Akademisierung von Heilmittelberufen gefordert. Bisher gibt es noch wenig empirische Erkenntnis dazu, wie sich dadurch die Versorgungsqualität der Patienten verändert hat [39].

Leitlinien geben Orientierungshilfen

Entscheidend für Versorgungsqualität und Patientensicherheit sind verlässliche Standards. Leitlinien setzen Qualitätsstandards und geben Orientierungshilfen - für die
Neurorehabilitation stehen sie in umfangreicher Anzahl zur Verfügung. Sie werden meist von Fachgesellschaften für die Praxis erarbeitet. Relevante Leitlinien stellen beispielsweise die Deutsche Gesellschaft für Neurorehabilitation, die European Federation of Neurological Societies, die Deutsche Gesellschaft für Neurotraumatologie und Klinische Neuropsychologie sowie die Deutsche Gesellschaft für Neurologie zur Verfügung. Die AWMF (Arbeitsgemeinschaft der Wissenschaftlichen Medizinischen Fachgesellschaften) definiert zudem verschiedene Entwicklungsstufen von Leitlinien, die die methodischen Anforderungen widerspiegeln: S1 bis S3, wobei S3 für die höchste methodische Fundierung steht. Die meisten bei der AWMF verfügbaren Leitlinien sind S1-Leitlinien [26]. Leitlinienkonforme Behandlung kann neben der gesicherten Behandlungsqualität auch zur besseren Vergleichbarkeit von Forschungsergebnissen beitragen.

Qualitätsstandards setzt auch die Bundesarbeitsgemeinschaft für Rehabilitation (BAR), die alle Leistungsträger der Rehabilitation vertritt. Wichtig für den Bereich Neurorehabilitation sind die Rahmenempfehlungen zur ambulanten neurologischen Rehabilitation, die Empfehlungen zur Neurologischen Rehabilitation von Patienten mit schweren und schwersten Hirnschädigungen in den Phasen B und C und die Empfehlungen zur stationären Langzeitpflege und Behandlung von Menschen mit schweren und schwersten Schädigungen des Nervensystems in der Phase F $[2,3,4]$.

Eine Übersicht zu Leitlinien für
die Neurorehabilitation bietet
die Arbeitsgemeinschaft der
Wissenschaftlichen Medizinischen
Fachgesellschaften (AWMF) unter
www.awmf.org.
Die Rahmenempfehlungen der
Bundesarbeitsgemeinschaft für
Rehabilitation (BAR) können unter
www.bar-frankfurt.de heruntergeladen
werden.

\section{Versorgungsepidemiologie}

Die Versorgungsepidemiologie analysiert die Einflüsse der einzelnen Merkmale des Versorgungssystems (Strukturen, Prozesse, Technologien, Leistungen) auf das Outcome der Versorgung, wie z.B. die Gesundheit und
Lebensqualität der Behandelten. Auch die Effectiveness-Forschung - die Untersuchung der Wirksamkeit evidenzbasierter Behandlungsmethoden unter Alltagsbedingungen ist hier einzuordnen [32].

Versorgungsepidemiologie der Neurorehabilitation

Versorgungsepidemiologischen Fragen widmete sich das Projekt Collaborative Evaluation of Rehabilitation in Stroke across Europe (CERISE). In vier europäischen Ländern wurde stationäre Schlaganfallrehabilitation hinsichtlich Outcome und Versorgungssystem verglichen, um Rückschlüsse auf günstige Rehabilitationsbedingungen zu ziehen. Ein Ergebnis waren positive Zusammenhänge zwischen der Therapiezeit der Patienten pro Tag und den motorisch-funktionellen Verbesserungen. Die Einrichtungen mit der längsten täglichen Therapiezeit sowie den größten motorisch-funktionellen Erfolgen lagen in der Schweiz und Deutschland, verglichen wurde mit einem belgischen und einem britischen Zentrum. Auf die Prävalenz von Angst und Depression nach einem Schlaganfall schienen die Unterschiede der Rehaeinrichtungen sich hingegen nicht auszuwirken [17].

Es wird deutlich, dass die Outcome-Kriterien der Neurorehabilitation sehr vielseitig sind. Graf und Kollegen greifen motorische und psychische Rehabilitationsergebnisse auf, das Spektrum möglicher OutcomeKriterien ist jedoch noch wesentlich breiter. Hinsichtlich Aktivitäten und Teilhabe sind berufliche und soziale Marker, aber auch z. B. Fähigkeiten einer sinnerfüllenden Freizeitgestaltung relevant. Differenzierte Kriterien aller dieser Bereiche sind notwendig, um Verbesserungen der äußerst heterogenen Schädigungen, Beeinträchtigungen und Behinderungen bei neurologischen Erkrankungen abbilden zu können. Einerseits ist wünschenswert, vielfältige Outcome-Kriterien zu berücksichtigen, andererseits ist für die Vergleichbarkeit von Studien ein Konsens über hauptsächlich relevante Ergebnisse erforderlich.

Farin setzt sich mit der Teilhabe, einem Outcome-Bereich von hoher gesundheitspolitischer Relevanz, auseinander [13]. Er kommt zu dem Schluss, dass das Teilhabekonzept der ICF insgesamt noch unzureichend operationalisiert ist und es an geeigneten Instrumenten mangelt, die Teilhabe präzise erfassen und einer Beforschung 
ausreichend zugänglich machen. Die Patientenorientierung der Rehabilitation ist ihm ebenfalls ein Anliegen. Farin und sein Team berichten hohe Ausschlussquoten neurologisch Erkrankter bei Patientenbefragungen in Qualitätssicherungsprogrammen [14]. Patientenorientierung in der Neurorehabilitation muss deshalb weiter gefasst werden und heißt, sich auf die teils begrenzte Mitwirkungsfähigkeit der Rehabilitanden und Rehabilitandinnen einzustellen. Dies erfordert eigene Wege, um den Patientenbedürfnissen trotz der individuell sehr verschiedenen Beeinträchtigungen gerecht zu werden.

\section{Fazit}

Zusammenfassend kann gesagt werden, dass in allen vorgestellten Teildisziplinen der Versorgungsforschung Erkenntnisse über die Versorgungssituation in der neurologischen Rehabilitation vorliegen. Es steht zumindest begrenzt Wissen zur Verfügung, beispielsweise über die Wirksamkeit einzelner Behandlungskonzepte, aber auch über Versorgungsdefizite. Damit kann bereits Veränderungsbedarf begründet und politisch vertreten werden, um eine bessere Gesundheitsversorgung zu erreichen. Auch Denkanstöße und neue Ansätze zur Verbesserung der Versorgung sind entstanden. Einige vorhandene Entwicklungen erscheinen durchaus positiv. Dennoch ist zentrales Ergebnis dieser Analyse im Bereich der Neurorehabilitation, dass in allen Disziplinen der Versorgungsforschung ein ausgeprägter Bedarf an weiterer Forschung besteht.

Ein Problem ist derzeit, dass zwar wissenschaftliche Erkenntnisse vorhanden sind, diese jedoch häufig aufgestellten methodischen Kriterien nicht genügen und somit beispielweise aus HTA-Berichten ausgeschlossen werden. Teils werden aufgrund dessen keinerlei Empfehlungen abgeleitet. Damit die Versorgungsforschung jedoch zu einer optimalen Ressourcennutzung beitragen kann, muss - mit David L. Sackett gesprochen - mit der „bestverfügbaren externen Evidenz" gearbeitet werden [38]. Das geschieht, wenn vorhandenes Wissen niedrigerer Evidenzstufen für die praktische $\mathrm{Ar}$ beit nutzbar gemacht und gleichzeitig eine methodisch hochwertige Versorgungsforschung vorangetrieben wird.

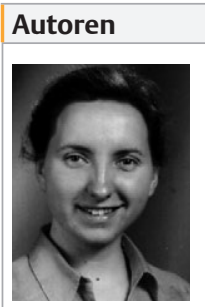

Angela Franke ist Rehabilitationspsychologin B. Sc. und Ergotherapeutin in Dresden. Von 2008 bis 2010 war sie in der Helene-Maier-Stiftung Kreischa mit den Schwerpunkten arbeitsbezogene Diagnostik und Therapie von

Menschen mit erworbenen Hirnschädigungen tätig. Derzeit schließt sie ihr Masterstudium der Rehabilitationspsychologie an der Hochschule Magdeburg-Stendal ab.

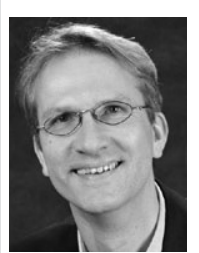

Prof. Dr. Matthias Morfeld ist Soziologe und Gesundheitswissenschaftler. Gegenwärtig hat er eine Professur für System der Rehabilitation an der Hochschule Magdeburg-Stendal.

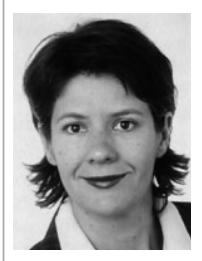

Prof. Dr. Claudia Wendel ist klinische Neuropsychologin und psychologische Psychotherapeutin. Seit 2004 unterrichtet sie an der Hochschule Magdeburg-Stendal und ist am Zentrum für ambulante Neuropsychologie und

Verhaltenstherapie in Berlin beschäftigt.

\section{Prof. Dr. Claudia Wendel}

Hochschule Magdeburg-Stendal (FH)

Osterburger Straße 25, 39576 Stendal

E-Mail: claudia.wendel@hs-magdeburg.de

\section{Bibliografie}

DOI 10.1055/s-0031-1287634

neuroreha 2011; 3: 107-113

(c) Georg Thieme Verlag KG

Stuttgart · New York · ISSN 1611-6496

\section{Literatur}

1. Andersen RM. Revisiting the Behavioral Model and Access to Medical Care: Does It Matter? J Health Soc Behav 1995; 36: 1-10

2. BAR Bundesarbeitsgemeinschaft für Rehabilitation. Empfehlungen zur Neurologischen Rehabilitation von Patienten mit schweren und schwersten Hirnschädigungen in den Phasen B und C. Frankfurt: BAR; 1999 http://www.bar-frankfurt.de/upload/Rahmenempfehlung_neuroligische_Reha_Phasen_B_und_C_149.pdf, 30.03.2011

3. BAR Bundesarbeitsgemeinschaft für Rehabilitation. Empfehlungen zur stationären Langzeitpflege und Behandlung von Menschen mit schweren und schwersten Schädigungen des Nervensystems in der Phase F. Frankfurt: BAR; 2003 http://www.bar-frankfurt. de/upload/Rahmenempfehlung_station\%C3\%A4re_ Langzeitpflege_151.pdf, 30.03.2011

4. BAR Bundesarbeitsgemeinschaft für Rehabilitation. Rahmenempfehlungen zur ambulanten neurologischen Rehabilitation. Frankfurt: BAR; 2005 http:/ www.bar-frankfurt.de/upload/Rahmenempfehlung_ neurologische_Rehabilitation_148.pdf, 30.03.2011
5. Bölsche F, Hasenbein U, Reißberg H et al. Ergebnisse der ambulanten und stationären Rehabilitation in den ersten sechs Monaten nach Schlaganfall. Fortschr Neurol Pychiat 2003; 71: 458-468

6. BMBF Bundesministerium für Bildung und Forschung Von der Forschung in die Versorgung Kompetenznetze in der Medizin. Bonn: BMBF; 2006 http://www. kompetenznetze-medizin.de/Infomaterial.aspx, 24.03.2011

7. Chan L, Wang H, Terdiman J et al. Disparities in outpatient and home health service utilization following stroke: Results of a 9-year cohort study in Northern California. PM \& R 2009; 11: 997-1003

8. Clare L, Woods B. Cognitive rehabilitation and cognitive training for early-stage Alzheimer's disease and vascular dementia. Cochrane Database of Systematic Reviews 2003; 4

9. DGNR Deutsche Gesellschaft für Neurorehabilitation. Diskussion: Qualitätsanforderungen und Vergütungssätze nach BAR Rahmenempfehlung. DGNR; 2010. http://www.dgnr.de/Qualitaetsanforderungen-undVerguetungssaet.4531.html, 30.03.2011

10. DIMDI Deutsches Institut für Medizinische Dokumentation und Information. Basisinformation: HTA beim DIMDI. http://www.dimdi.de/static/de/hta/basisinfo_de.pdf, 14.03.2011

11. Ehret R, Balzer-Geldsetzer M, Reese JP et al. Direkte Kosten der PMarkinson-Behandlung: Eine Erhebung in neurologischen Schwerpunktpraxen in Berlin. Nervenarzt 2009; 80: 452-458

12. EUnetHTA European Network for Health Technology Assessment. About. http://www.eunethta.net/Public/ About_EUnetHTA/, 30.03.2011

13. Farin E. Teilhabe von Patienten an Lebensbereichen als Gegenstand der Versorgungsforschung: Beziehung zu verwandten Konstrukten und Übersicht über vorhandene Messverfahren. Gesundheitswesen 2011; 73: 1-11

14. Farin E, Jäckel WH, Schalaster V. Das Qualitätssicherungsverfahren der GKV in der medizinischen Rehabilitation: Ergebnisse und Weiterentwicklung. Gesundheitswesen 2009; 71: 163-174

15. Frank W, Konta B. HTA-Bericht 26: Kognitives Training bei Demenzen und andere Störungen mit kognitiven Defiziten Köln: DIMDI; 2005 http://portal.dimdi.de/de/hta/hta_berichte/hta123_bericht_de.pdf, 29.03.2011

16. Gerdes N, Funke UN, Schüwer U et al. Ergebnisorientierte Vergütung der Rehabilitation nach Schlaganfall - Entwicklungsschritte eines Modellprojekts 2001-2008. Rehabilitation 2009; 48: 190-201

17. Graf J, Schupp W, De Wit L et al. Gemeinschaftliche Evaluation der Schlaganfallrehabilitation innerhalb von Europa - Klinische Aspekte. Neurol Rehabil 2009; 15: 275-289

18. Heel S, Fischer S, Fischer S et al. Versorgungsforschung in der Klinischen Neuropsychologie - eine Standortbestimmung. Z Neuropsychol 2008; 19: 253-269

19. Heuschmann PU, Busse O, Wagner M et al. Schlaganfallhäufigkeit und Versorgung von Schlaganfallpatienten in Deutschland. Akt Neurol 2010; 37: 333-340

20. Hoffmann T, Bennett S, Koh CL et al. Occupational therapy for cognitive impairment in stroke patients. Cochrane Database of Systematic Reviews 2010; 9

21. IOM Institute of Medicine. Crossing the Quality Chasm. A New Health System for the 21st Century. Washington: National Academy Press; 2001 http://www.nap.edu/books/0309072808/html/, 23.03.2011

22. Jäckel WH. Qualität in der Rehabilitation. Rehabilitation 2010; 49: 345-355

Das Literaturverzeichnis zu diesem Artikel ist hier nicht komplett abgedruckt. Die vollständige Version finden Sie im Internet unter www.thieme-connect.de/ ejournals/toc/neuroreha. 